\title{
Initiation of Impulsively Fast Magnetic Reconnection Induced by Current Sheet Ejection*)
}

\author{
Michiaki INOMOTO, Shuji KAMIO, Akihiro KUWAHATA ${ }^{1)}$, Takenori G. WATANABE, \\ Kotaro YAMASAKI, Kazutake KADOWAKI, Taichi ITO ${ }^{1)}$, Yasuhiro KAMINOU, Takuma YAMADA \\ and Yasushi ONO \\ Graduate School of Frontier Sciences, The University of Tokyo, Kashiwa, Chiba 277-8561, Japan \\ ${ }^{1)}$ Graduate School of Engineering, The University of Tokyo, Bunkyo, Tokyo 113-8656, Japan
}

(Received 26 November 2012 / Accepted 14 June 2013)

\begin{abstract}
Fast magnetic reconnection mechanism triggered by current sheet ejection is studied in plasma merging laboratory experiment with a strong guide field. The current sheet is hardly compressed in the presence of the guide field and slow steady reconnection evolves in the early phase. It then transits to impulsively fast magnetic reconnection when the current sheet is ejected from the $\mathrm{X}$ point region. Behavior of the current sheet is investigated in detail by comparison with the fast reconnection case provided by anomalous resistivity.
\end{abstract}

(c) 2013 The Japan Society of Plasma Science and Nuclear Fusion Research

Keywords: magnetic reconnection, current sheet, plasma merging, laboratory experiment

DOI: $10.1585 / \mathrm{pfr} .8 .2401112$

\section{Introduction}

Magnetic reconnection [1,2] is an important elementary process in a broad range of magnetized plasmas, such as solar atmosphere, magnetosphere, astrophysical objects and fusion plasmas. Magnetic reconnection provides variety of global events; magnetic self-organization, formation of jet structure, rapid conversion from magnetic to kinetic/thermal energy, and so on. Magnetic reconnection is usually formulated as a steady MHD phenomenon, but the simple model suggested by Sweet [3] and Parker [4] requires large enhancement of plasma resistivity inside the diffusion region to establish fast magnetic reconnection observed in the universe. Another steady model proposed by Petschek [5] provides faster magnetic reconnection than the previous one, although local enhancement of resistivity is necessary [6] for sustaining the slow shock structure which yields efficient conversion from magnetic to kinetic energy. Nevertheless, observed reconnection events are not always steady phenomena. They often show impulsive or intermittent nature, as seen in solar flare [7] and sawtooth oscillation [8]. One candidate for the impulsive reconnection is provided by a growth of plasmoid [9] or current sheet and its ejection.

In this paper, the experimentally observed impulsive reconnection due to the current sheet ejection [10] is investigated by using detailed magnetic measurement. The sheet current density takes its maximal value at the $\mathrm{X}$ point in steady magnetic reconnection, but more dynamic reconnection process evolves non-steadily in plasma merging experiment under strong guide field. In such case the

author's e-mail: inomoto@k.u-tokyo.ac.jp

*) This article is based on the presentation at the 22nd International Toki Conference (ITC22). plasma inflow and outflow do not always match and the sheet current could temporarily move away from the $\mathrm{X}$ point as if it were ejected from the $\mathrm{X}$ point. The behavior of the current sheet in the case of current sheet ejection is compared with that in the case of steady fast reconnection caused by anomalous resistivity.

\section{Experimental Setup}

The TS-3 [11] device (Fig. 1) can generate two magnetically confined plasma toroids with both toroidal and poloidal magnetic fields induced by discharge between the electrodes (z-discharge) and swing down of the poloidal field (PF) coil currents ( $\theta$-pinch). In this research, cohelicity merging scheme [12] is employed, in which the poloidal magnetic fields in both plasma toroids reconnect in the $r-z$ plane and the toroidal magnetic field perpendicular to the reconnection plane remains as a guide magnetic field. The magnitude of the guide magnetic field can be controlled by changing the center conductor current $I_{z}$ flowing along the geometric axis. Reconnection inflow is externally driven by the reversed PF coil current which pushes the two plasma toroids to approach each other.

Magnetic field structure around the $\mathrm{X}$ point region is observed by a two dimensional pickup coil array which has the minimum resolution of $1.5 \mathrm{~cm}$ in $z$ (current sheet thickness) direction. Poloidal magnetic flux $\Psi$, toroidal current density $j_{\mathrm{t}}$ and toroidal electric field $E_{\mathrm{t}}$ are calculated from the obtained magnetic field data. Electron temperature and density are measured by a Langmuir probe and ion temperature is measured by a Doppler spectroscopic measurement. Typical plasma parameters in hydrogen discharge are; electron temperature $T_{\mathrm{e}}=5-10 \mathrm{eV}$, ion temperature 


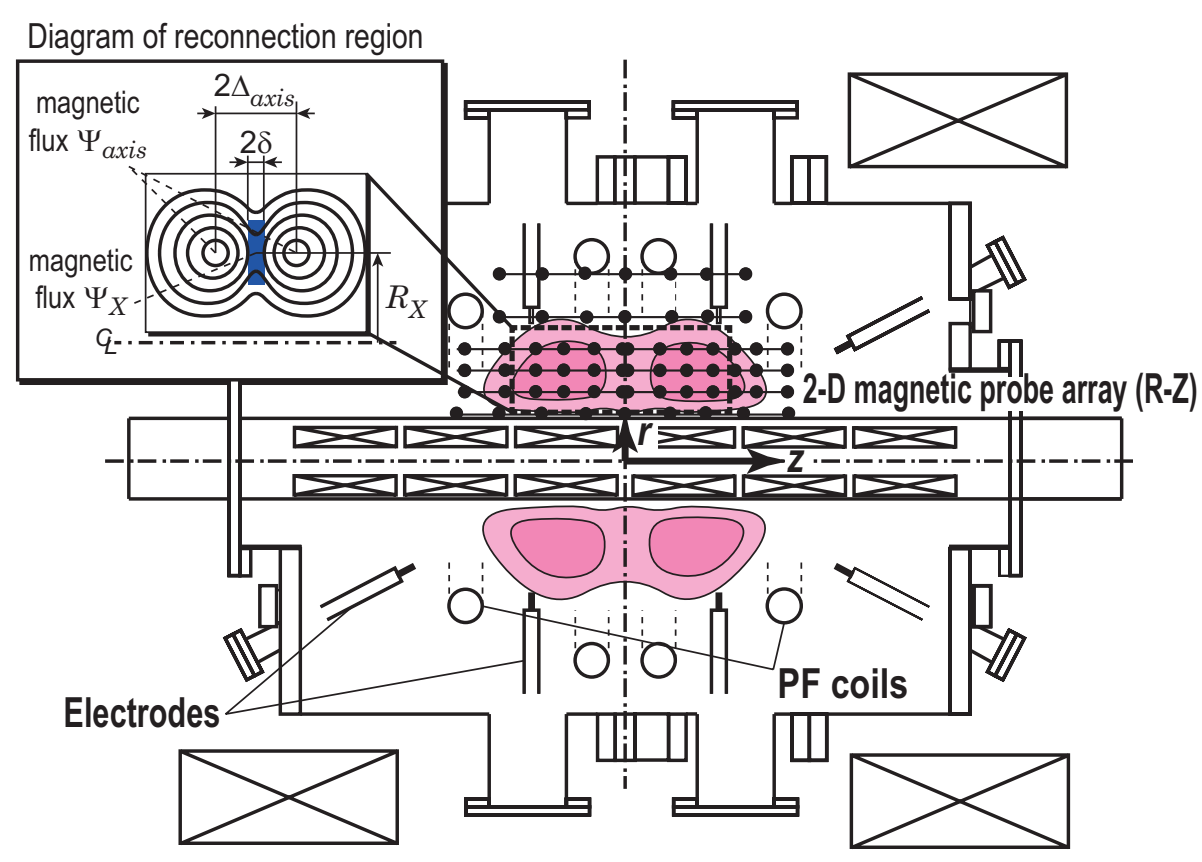

Fig. 1 Schematic view of the TS-3 plasma merging experiment.
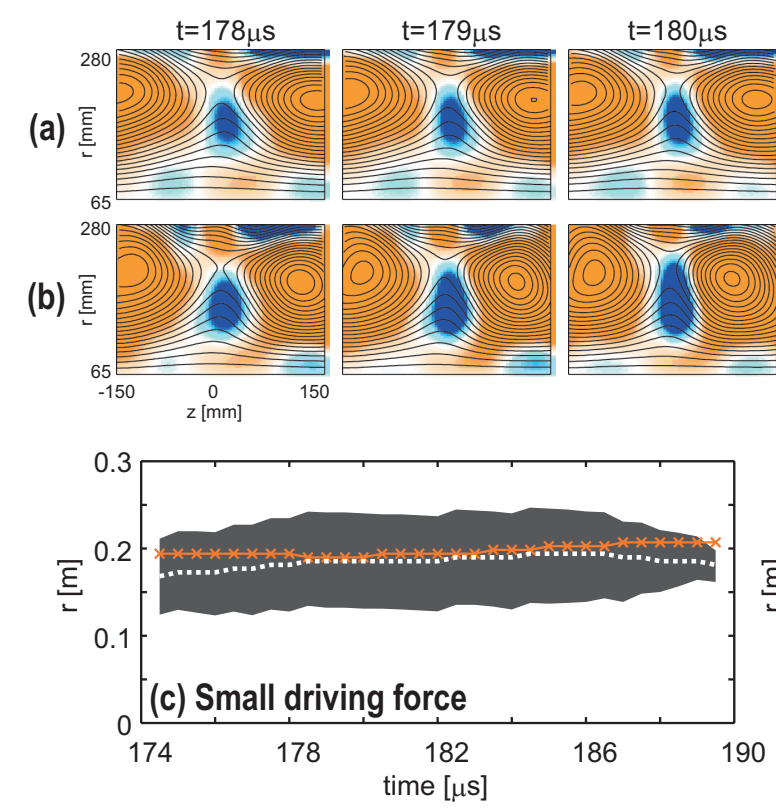
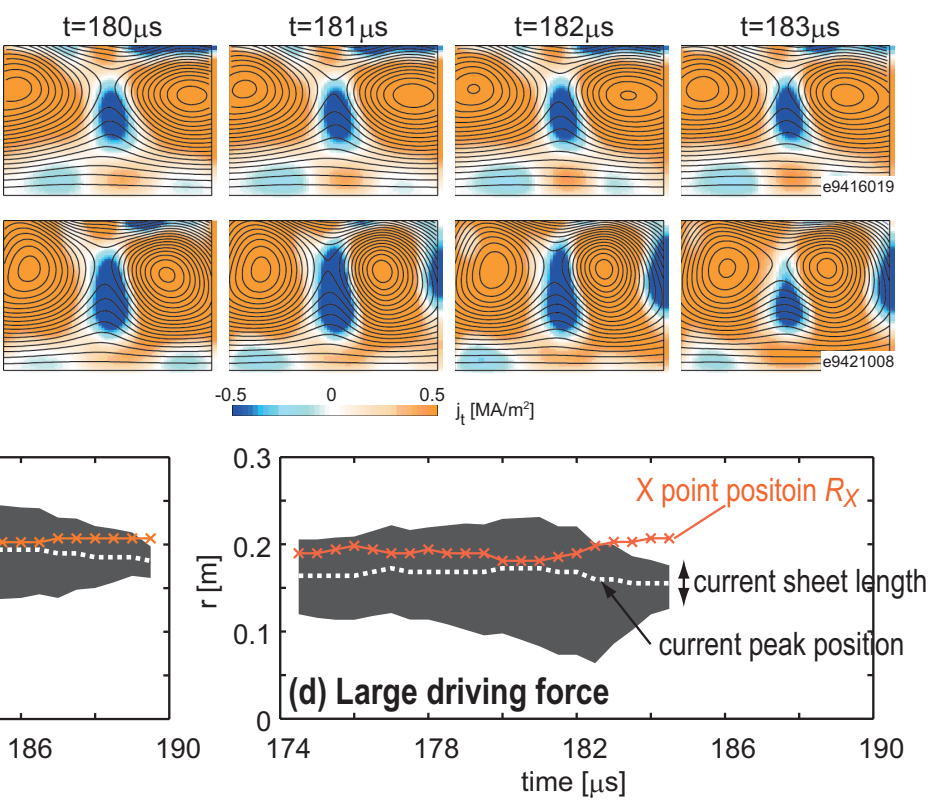

Fig. 2 Time evolutions of reconnecting field lines (contour spacing : $0.3 \mathrm{mWb}$ ) and toroidal current density (color) in the presence of a strong guide field with (a) small and (b) large external driving force. (c) and (d) show the evolutions of current sheet shape, radial locations of the $\mathrm{X}$ point and the current peak.

$T_{\mathrm{i}}=5-50 \mathrm{eV}$, electron density $n_{\mathrm{e}}=5-10 \times 10^{19} \mathrm{~m}^{-3}$.

\section{Experimental Results and Discus- sion}

Figures 2 (a) and (b) show evolutions of two dimensional magnetic flux surfaces ( $\Psi$ contours with spacing of $0.3 \mathrm{mWb}$ ) around the $\mathrm{X}$ point region of the magnetic reconnection in the presence of strong guide field. The initial (vacuum) guide field $B_{\mathrm{t}}$ applied by the center conduc- tor current $I_{z}$ is about $40 \mathrm{mT}$, which is four times as high as the typical reconnection magnetic field of about $10 \mathrm{mT}$. The toroidal (out-of-plane) current density is superposed on Figs. 2 (a) and (b) by color. When the external force pushing the two merging plasma toroids is small, the magnetic reconnection develops steadily with small reconnection rate $M_{\text {in }}=E_{\mathrm{t}, \mathrm{X}} / B_{\mathrm{r}, \text { up }} V_{\mathrm{A}} \sim 0.1$, as shown in Fig. 2 (a). Here, $E_{\mathrm{t}, \mathrm{X}}$ is the reconncetion electric field at the $\mathrm{X}$ point, $B_{\mathrm{r} \text {,up }}$ is the reconnection magnetic field measured at the shoulder of the current sheet, and $V_{\mathrm{A}}$ is the Alfvén velocity. 

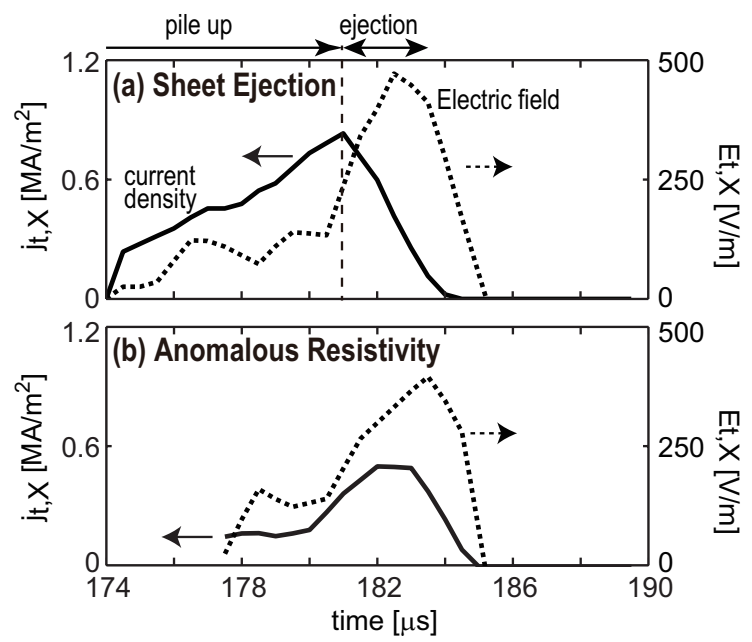

Fig. 3 Time evolutions of toroidal current density $j_{\mathrm{t}, \mathrm{X}}$ and toroidal electric field $E_{\mathrm{t}, \mathrm{X}}$ measured at the X point during fast reconnection due to (a) current sheet ejection and (b) anomalous resistivity.

The effective resistivity $\eta_{\mathrm{eff}}=E_{\mathrm{t}, \mathrm{X}} / j_{\mathrm{t}, \mathrm{X}}$ keeps small value of about $0.1 \mathrm{~m} \Omega \mathrm{m}$, which is twice as high as the classical parallel Spritzer resistivity. On the other hand, much faster reconnection condition is achieved when large external driving force is applied. In this situation, the current sheet once grows in length and moves toward inboard side as if it were crowded out from the $\mathrm{X}$ point, as shown in Fig. 2 (b). This kind of event is observed in TS-3 and other reconnection experiments under the condition of strong guide field and large external driving force.

Figures 2 (c) and (d) show the time evolutions of current sheet radial shape (gray area), radial locations of $\mathrm{X}$ point $R_{\mathrm{X}}$ (cross) and current peak (dotted line). The small driving force case shows steady evolution of the current sheet which involves the $\mathrm{X}$ point in it in the whole reconnection period. While the current sheet gradually increases its length in the case with large external driving force and starts to move towards inboard side at $t=181 \mu \mathrm{s}$. The $\mathrm{X}$ point is then excluded from the current sheet region. This event is interpreted as a 'current sheet ejection' [10] which takes place after the 'pile-up' [8] of the magnetic flux in the upstream region when the extremely driven inflow flux exceeds the outflow flux determined by the magnetic reconnection rate. The sheet current prevents the magnetic field lines approaching the $\mathrm{X}$ point and its sudden disappearance due to the sheet ejection will induce large enhancement of the reconnection inflow, leading to an impulsive fast magnetic reconnection like a plasmoid ejection case [9].

In the TS-3 plasma merging experiment, another fast reconnection condition is achieved by some kind of micro instability relevant to the ion's kinetic effect. Anomalous enhancement of the effective resistivity was observed when the current sheet width is compressed shorter than the ion gyro radius [11], indicating that disturbance of the ion meandering motion inside the sheet current will cause anomalous dissipation of the current sheet [13]. To clarify the fast magnetic reconnection mechanism induced by the current sheet ejection, we will compare the sheet ejection case (with large guide field and large external driving force) with the anomalous resistivity case (with small guide field and large external driving force) [11].

Figure 3 shows the time evolutions of toroidal current density $j_{\mathrm{t}, \mathrm{X}}$ and reconnection electric field $E_{\mathrm{t}, \mathrm{X}}$ measured at the $\mathrm{X}$ point. In the current sheet ejection case, sudden enhancement of the reconnection electric field takes place concurrently with the sheet ejection at $t=181 \mu \mathrm{s}$ after a slow reconnection period with gradually increasing current density. On the contrary, the current density and the reconnection electric field in the anomalous resistivity case evolve mostly in phase, as shown in Fig. 3 (b). Under the assumption that the current sheet structure is radially uniform, the toroidal current density at the $\mathrm{X}$ point is approximately expressed as

$$
j_{\mathrm{t}, \mathrm{X}} \sim \frac{1}{\mu_{0}} \frac{\partial B_{\mathrm{r}}}{\partial z} \sim \frac{B_{\mathrm{r}, \mathrm{up}}}{\mu_{0} \delta},
$$

where $\delta$ is the current sheet half width. The reconnection magnetic field $B_{\text {r,up }}$ is also expressed using the poloidal magnetic flux $\Psi_{\text {axis }}$ at the axis of the merging toroid, the poloidal magnetic flux $\Psi_{\mathrm{X}}$ at the $\mathrm{X}$ point, and the distance $2 \triangle_{\text {axis }}$ between the two magnetic axes of the merging toroids (these definitions are illustrated in upper-left part of Fig. 1) as

$$
B_{\mathrm{r} \text {,up }}=-\frac{1}{2 \pi r} \frac{\partial \Psi}{\partial z} \sim \frac{\Psi_{\mathrm{axis}}-\Psi_{\mathrm{X}}}{2 \pi R_{\mathrm{X}}\left(\Delta_{\mathrm{axis}}-\delta\right)} .
$$

Thus the current density at the $\mathrm{X}$ point is given by the following formula

$$
j_{\mathrm{t}, \mathrm{X}} \sim \frac{\Psi_{\mathrm{axis}}-\Psi_{\mathrm{X}}}{2 \pi R_{\mathrm{X}} \mu_{0}} \frac{1}{\delta\left(\Delta_{\mathrm{axis}}-\delta\right)} .
$$

The current density is inversely proportional to the current sheet width $\delta$ and the distance between the current sheet edge and the magnetic axis of the merging toroid $\left(\Delta_{\text {axis }}\right.$ $\delta$ ), hence the increase in current density will be provided either by thinning of the current sheet (decreasing $\delta$ ) or by compression in the upstream region (decreasing $\left(\Delta_{\text {axis }}-\delta\right)$ ).

Figure 4 (a) shows the evolutions of the current sheet width $2 \delta$ across the $\mathrm{X}$ point and the distance $2 \Delta_{\text {axis }}$ between the two magnetic axes of the merging plasma toroids for the fast reconnection by current sheet ejection. The current sheet width takes nearly constant value of $2 \delta \sim 7 \mathrm{~cm}$ during the pile-up phase, although the distance $2 \Delta$ axis between the magnetic axes shows rapid decrement. Thus, the current sheet in the current sheet ejection case is hardly compressed and the increase in the toroidal current density is mostly provided by the compression in the upstream region, i.e. pile-up of the inflow magnetic flux. The current sheet half width of $\delta \sim 3.5 \mathrm{~cm}$ during the 


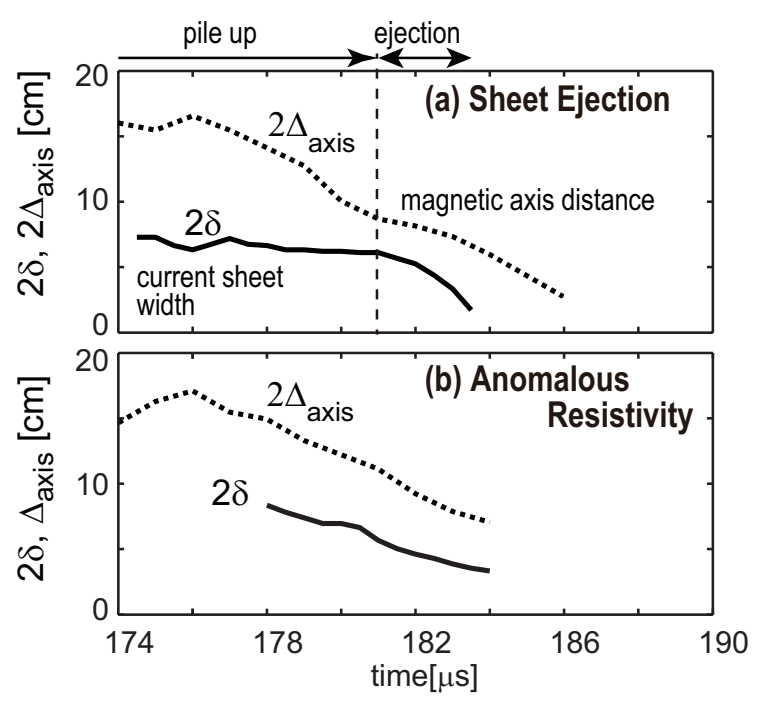

Fig. 4 Time evolutions of current sheet width $2 \delta$ and distance $2 \Delta_{\text {axis }}$ between magnetic axes of the merging plasma toroids during fast reconnection due to (a) current sheet ejection and (b) anomalous resistivity.

pile-up phase is much longer than the local ion gyroradius of about $0.5 \mathrm{~cm}$, or the Sweet-Parker layer thickness $\delta_{\mathrm{SP}}=L / S^{1 / 2} \sim 1.5 \mathrm{~cm}$, where $L$ is the system size and $S$ is the Lundquist number. The initial half width of the current sheet is more close to the ion inertia length $c / \omega_{\mathrm{pi}}=2.4$ $3.2 \mathrm{~cm}$, indicating that the observed current sheet is the ion current sheet in the collisionless reconnection condition with strong guide field. The anomalous resistivity case shown in Fig. 4 (b) shows a sharp contrast with the current sheet ejection case. The slope of the current sheet width is close to that of the magnetic axes distance; hence the increment of the current density in the anomalous resistivity case is mainly provided by the thinning of the current sheet.

Figure 5 shows the time evolutions of the reconnection magnetic field $B_{\mathrm{r} \text {,up }}$ measured at the shoulder of the current sheet, the guide magnetic field $B_{\mathrm{t}, \mathrm{X}}$ at the X point, and the reconnection rate $M_{\text {in }}$. Significant increment of the reconnection magnetic field is observed during the pileup phase of the current sheet ejection case because of the strong compression in the upstream region. The anomalous resistivity case, however, shows moderate increment of the reconnection magnetic field during the sheet compression. The difference in the current sheet compressibility is possibly due to the large difference in the guide magnetic field, as shown in Fig. 5 (b). The strong guide field in the current sheet ejection case will sustain the constant current sheet width against the large inflow driven by the external force.

In the current sheet ejection case, the rushing inflow magnetic flux piles up in the upstream region to modify the local curvature of the magnetic field lines near the current sheet. The current sheet shape is then radially elongated, as shown in Fig. 2 (d), and when the sheet extends into the

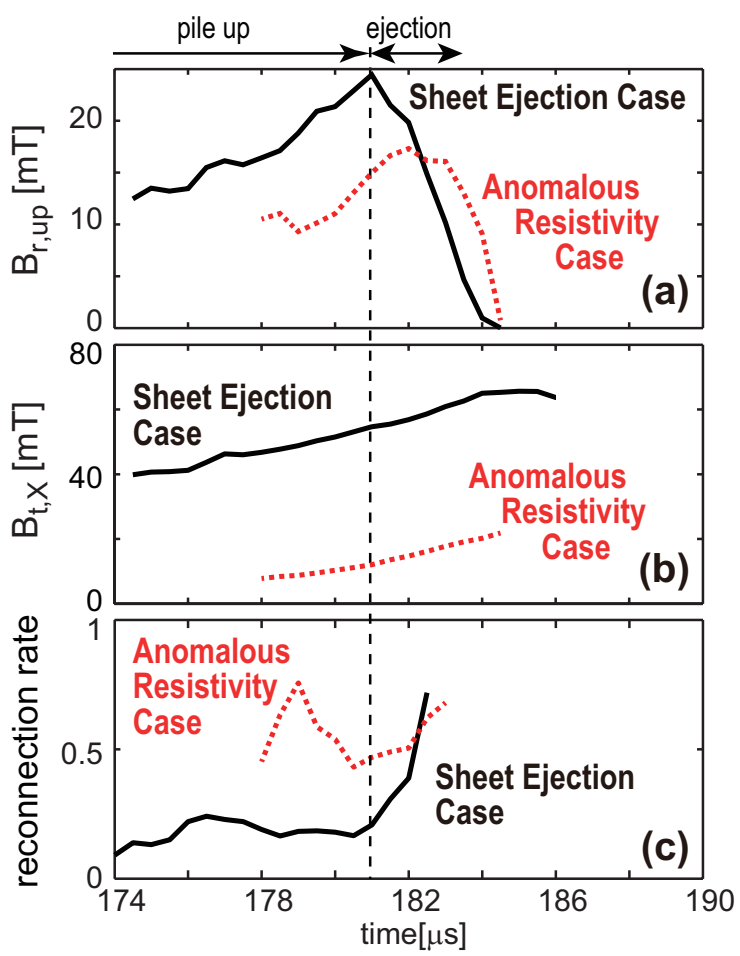

Fig. 5 Time evolutions of (a) reconnection magnetic field $B_{\text {r,up }}$ at the shoulder of the current sheet, (b) toroidal (guide) magnetic field $B_{\mathrm{t}, \mathrm{X}}$ at the $\mathrm{X}$ point and (c) reconnection rate during fast reconnection due to current sheet ejection and anomalous resistivity.

downstream region where the reconnected (axial) magnetic field is dominant, the sheet current becomes radially unstable and is ejected from the $\mathrm{X}$ point region. The anomalous resistivity case keeps high reconnection rate $M_{\text {in }}$ during the whole reconnection period, while the current sheet ejection case shows steep increase just after the current sheet ejection starts, indicating the onset of impulsively fast magnetic reconnection by the current sheet ejection.

\section{Summary}

An experimental observation of impulsively fast magnetic reconnection is presented. Collisionless magnetic reconnection in the presence of strong guide field starts as a slow steady reconnection with nearly constant current sheet width close to the ion inertia length. Large external force drives the inflow magnetic flux and increase the reconnection magnetic field by the 'pile-up' effect. The radially elongated current sheet will then become unstable against its radial motion and finally it is ejected from the $\mathrm{X}$ point region. This current sheet ejection drives large inflow impulsively to enhance the reconnection rate even in high magnetic Reynolds number regime. To evaluate the quantitative nature of the current-sheet-ejection-driven magnetic reconnection, further experimental studies are required to clarify its parametric dependencies on plasma and reconnection parameters as well as numerical studies to simulate 
the experimental conditions.

\section{Acknowledgments}

This work was supported in partial by Grant-in-Aid for Scientific Research (KAKENHI) 22246119, 22686085 and JSPS Core-to-Core Program 22001.

[1] M. Yamada, R. Kulsrud and H. Ji, Rev. Mod. Phys. 82, 603 (2010).

[2] E.G. Zweibel and M. Yamada, Annu. Rev. Astron. Astrophys. 47, 291 (2009).

[3] P.A. Sweet, in Electromagnetic Phenomenon in Cosmical Physics, edited by B. Lehnert (Cambridge University Press,
New York, 1958) vol.6, p.123.

[4] E. Parker, J. Geophys. Res. 62, 509 (1957).

[5] H.E. Petschek, NASA Spec. Pub. 50, 425 (1964).

[6] D.A. Uzdensky and R.M. Kulsrud, Phys. Plasmas 7, 4018 (2000).

[7] S. Masuda et al., Nature 371, 495 (1994).

[8] M. Yamada, Phys. Plasmas 18, 111212 (2011).

[9] K. Shibata and S. Tanuma, Earth Planets Space 53, 473 (2001).

[10] Y. Ono et al., Phys. Plasmas 18, 111213 (2011).

[11] Y. Ono et al., Phys. Plasmas 4, 1953 (1997).

[12] M. Yamada et al., Phys. Rev. Lett. 65, 721 (1990).

[13] R. Horiuchi and T. Sato, Phys. Plasmas 4, 277 (1997). 